Roland Bender · M. Azim Surani · Rashmi Kothary

Li-Lan Li · Dieter O. Fürst · Bodo Christ

Reinald Fundele

\title{
Tissue specific loss of proliferative capacity of parthenogenetic cells in fetal mouse chimeras
}

Received: 26 September 1994 / Accepted: 5 January 1995

\begin{abstract}
Parthenogenetic cells are lost from fetal chimeras. This may be due to decreased proliferative potential. To address this question, we have made use of combined cell lineage and cell proliferation analysis. Thus, the incorporation of bromodeoxyuridine in S-phase was determined for both parthenogenetic and normal cells in several tissues of fetal day 13 and 17 chimeras. A pronounced reduction of bromodesoxyuridine incorporation by parthenogenetic cells at both developmental stages was only observed in cartilage. In brain, skeletal muscle, heart and intestinal epithelium, this reduction was either less pronounced or observed only at one of the developmental stages analysed. No difference between parthenogenetic and normal cells was observed in epidermis and ganglia. Our results show that a loss of proliferative potential of parthenogenetic cells during fetal development contributes to their rapid elimination in some tissues. The analysis of the fate of parthenogenetic cells in skeletal muscle and cartilage development demonstrated different selection mechanisms in these tissues. In skeletal muscle, parthenogenetic cells were largely excluded
\end{abstract}

R. Bender ${ }^{1} \cdot$ R. Fundele $(-)$

Institut für Biologie III der Universität Freiburg,

Schänzlestrasse 1, D-79104 Freiburg, Germany

M. A. Surani $\cdot$ L-L. Li

Wellcome/CRC Institute of Cancer and Developmental Biology, Tennis Court Road, Cambridge CB2 1QR, and Physiological Laboratory, University of Cambridge, United Kingdom

R. Kothary

Institut du Cancer de Montréal, 1560 rue Sherbrooke est, Montréal, Québec H2L 4MI, Canada

D. O. Fürst

Max-Planck-Institut für Biophysikalische Chemie,

Am Fassberg, D-37077 Göttingen, Germany

B. Christ

Institut für Anatomie der Universität Freiburg, Albertstraße 17,

D-79104 Freiburg, Germany

Present address:

${ }_{1}^{1}$ Institut für Anatomie der Universität Freiburg, Albertstraße 17,

D-79104 Freiburg, Germany from the myogenic lineage proper by early post-midgestation. In primary hyaline cartilage, parthenogenetic cells persisted into adulthood but were lost from cartilages that undergo ossification during late fetal development.

Key words Parthenogenesis · Mouse chimeras . Proliferation · Differentiation

\section{Introduction}

The differential behaviour of maternally and paternally inherited alleles of imprinted genes is the cause of the lethality of parthenogenetic (pg) and androgenetic (ag) genotypes during postimplanation mouse development (reviewed by Solter 1988). However, both ag and pg embryos can be rescued for a study of their further developmental potential by the formation of chimeras with normal embryos (reviewed in Fundele and Surani 1994). Most studies were carried out on pg $<->$ fertilized (wt) chimeras, as pg embryos can be readily obtained by ethanol activation of ovulated oocytes (Kaufman 1978). These studies of chimeras containing a pg-derived cell lineage have shown that they exhibit highly specific and consistent phenotypes.

The growth of pg <-> wt chimeras is considerably delayed (Fundele and Surani 1994) and the degree of size reduction is positively correlated with the number of $\mathrm{pg}$ cells present in the chimera (Fundele et al. 1990). Chimeras with a high contribution of pg cells may have only $50 \%$ of the body weight of normal litter mates (Fundele et al. 1989, 1990). However, such chimeras do not survive prenatal or early postnatal development. Chimeras with only a minor pg contribution develop normally, apart from their reduced size, and when composed of two female cell lineages, are able to produce offspring derived from the pg lineage (Stevens 1978; Anderegg and Markert 1986; Nagy et al. 1989; Fundele et al. 1990). Studies of pg <-> wt chimeras have further demonstrated that pg cells suffer severe negative selection during fetal development. The temporal onset of selec- 
tion varies between different tissues and in some tissues selection may not occur at all. There is evidence that pg cells are allocated randomly to the different cell lineages of preimplantation pg $<->$ wt chimeras (Clarke et al. 1988; Thomson and Solter 1989). In late gastrulation stages, pg cells occur mainly in tissues derived from the inner cell mass and are absent from the trophoblast (Clarke et al. 1988; Thomson and Solter 1988). The loss of pg cells from the trophoblast is the first instance of the selection against them. In later stages of development selection against pg cells becomes apparent in most other tissues and is most pronounced in skeletal muscle, pancreas and liver (Fundele et al. 1990). In newborn pg <-> wt chimeras, pg-derived cells are generally no longer observed in these tissues.

In the present study we provide, for the first time, information on the cellular distribution of pg cells in fetal chimeras and the proliferation rates of pg and wt cells in several tissues of these chimeras. In addition, we can show that the mechanisms that are involved in the selection against pg cells differ between tissues.

\section{Materials and methods}

\section{Animals}

Outbred albino CFLP (originally from Bantin and Kingman stock) and NMRI (from the Zentralinstitut für Versuchstierzucht, Hanno-

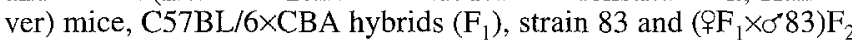
hybrid mice were used. Strain 83 mice are homozygous for a high copy transgenic insertion of a plasmid containing a mouse $\beta$-globin gene with flanking plasmid pBR322 sequences (Lo 1986).

\section{Embryos and chimeras}

Superovulation of females, collection, activation and handling of eggs and embryos, and embryo aggregation were all carried out according to standard procedures previously described (Surani et al. 1988). Pg $\leftrightarrow>$ wt chimeras were prepared by asynchronous aggregations with a pg embryo approximately $16 \mathrm{~h}$ more advanced than the fertilized embryo. Aggregated embryos were transferred into the uterine horns of recipient females on day 3 of pseudopregnancy. The day of formation of the vaginal plug of the recipient female was always counted as the first day of gestation. For identification of chimeric fetuses and adults, tissue processing, and analysis of transgenic contribution see Jägerbauer et al. (1992). For cell proliferation studies, pregnant recipient females were injected intraperitoneally with $50 \mathrm{mg}$ 5-bromo-2'-deoxyuridine (BrdU)/kg bodyweight approximately $1 \mathrm{~h}$ before sacrifice (Gratzner 1982).

Histology, immunohistochemistry (IHC) and in situ hybridization (ISH)

Embryos and adult tissues were fixed in Carnoy's solution containing $60 \%$ ethanol, $30 \%$ trichlormethane and $10 \%$ acetic acid, dehydrated and embedded in paraffin. For the detection of ossification a modification of a classic silver staining technique was applied (Kossa 1901). The following antibodies (ABs) were obtained from Dako Diagnostika, Hamburg: anti-BrdU, anti-fluorescein isothiocyanate (FITC) and alkaline phosphatase (AP), and peroxidase (POD)-conjugated second antibodies directed against mouse and rabbit immunoglobulins (Igs). A polyclonal $\mathrm{AB}$ directed against bovine Type II collagen that reacts with the mouse antigen (Sasano et al. 1992) was obtained from Biermann Diagnostica, Bad Nauheim and a polyclonal $A B$ raised in rabbit against desmin from Eurodiagnostics. Incubations with primary $\mathrm{ABs}$ were always carried out overnight at $4^{\circ} \mathrm{C}$, secondary $\mathrm{AB}$ reactions for 60 min at room temperature (RT). Dilutions of $A B s$ were in the range suggested by the suppliers.

For detection of Type II collagen, conventional indirect IHC was applied after hyaluronidase treatment of sections (Sasano et al. 1992). Either FITC, AP or POD-conjugated secondary ABs were used with hexazotized new fuchsin (NF) or 3,3'-diaminobenzidine (DAB) as chromogenic substrates.

For the detection of incorporated BrdU, sections were first treated with $25-50 \mu \mathrm{g}$ proteinase $\mathrm{K} / \mathrm{ml} 2 \times \mathrm{SSC}, 0.1 \%$ Triton $\mathrm{X}-100$ for $15-20 \mathrm{~min}$ at $37^{\circ} \mathrm{C}$ and refixed in $4 \%$ formaldehyde, $2.5 \%$ glutaraldehyde in $2 \times \mathrm{SSC}$. After protease treatment and fixation, DNA was denatured in $70 \mathrm{~mm} \mathrm{NaOH}$ for exactly 3 min. After $\mathrm{AB}$ reactions, BrdU-positive nuclei were stained using the AP reaction with NF as chromogenic substrate. Anti-BrdU IHC was always carried out subsequent to in situ hybridization; proteinase $\mathrm{K}$ digestion was omitted when it had been carried out prior to hybridization. DNA denaturation was performed both before hybridization and $\mathrm{BrdU}$ detection.

ISH directed against the transgenic insert was carried out essentially as described previously (Jägerbauer et al. 1992), using digoxigenin-labelled pMBG $\Delta 2$ as probe (Lo 1986; Lo et al. 1987).

For the detection of insulin-like growth factor II IGF2 mRNA, a 680 bp Hinfl-Pstl fragment from human IGF-II cDNA was subcloned into pGEM-3 vector. Transcription with SP6 RNA polymerase from a non linear recombinant plasmid generated the antisense probe; T7 RNA polymerase generated the sense probe. Both probes were labelled with ${ }^{35} \mathrm{~S}-U T \mathrm{TP}$. ISH was carried out as described in Wilkinson and Green (1990). Briefly, sections were treated with proteinase $\mathrm{K}(10 \mu \mathrm{g} / \mathrm{ml})$ and hybridized with the probe overnight at $55^{\circ} \mathrm{C}$. The sections were then subjected to a high stringency wash at $55^{\circ} \mathrm{C}$ for $30 \mathrm{~min}$. After dehydration, slides were coated with Ilford $\mathrm{K} 5$ emulsion and exposed at $4^{\circ} \mathrm{C}$ for 7 days. Developed slides were stained with haematoxylin and mounted in Kirkpatrick and Lendrum's DPX (Distyrene/Dibutyl phthalate/Xylene) DPX.

\section{Selection of tissues and evaluation of in situ hybridization}

Tissues selected for proliferation and/or quantitative analysis were epidermis, brain, dorsal root ganglia, cartilage, cardiac muscle, smooth muscle, mesenchyme and intestinal epithelium. These tissues were chosen in part for their suitability for quantitative evaluation; the nuclear number in dorsal root ganglia, epidermis, cartilage and heart can easily be counted. This may either be due to cell size or, in the case of epidermis, to the regular spatial arrangement of cells. However, brain and gut epithelium were selected for analysis even though they are difficult to count. The areas of any given tissue that were counted were selected randomly.

For the quantitation of $\mathrm{pg}$ contribution to $\mathrm{pg}\langle->$ wt chimeras and $\mathrm{pg}$ and wt proliferation rates, at least 2 different areas from each tissue were analysed. For cartilage, between 5 and 24 different areas were analysed due to the small size of the individual cartilages. Pictures of tissues were taken on a Zeiss Axiophot microscope with Ektachrome EHC 100 film. Slides were projected against a screen. Total numbers of nuclei stained with 4,6diamidino-2-phenylindole DAPI and nuclei labelled by ISH and/or anti-BrdU IHC were counted manually. The statistical significance of the differences between proliferation rates of $\mathrm{pg}$ and wt nuclei was calculated using the Cochran-Mantel-Haenszel test (Matthews and Farewell 1988).

\section{Results}

\section{Chimeras}

Thirteen fetal chimeras with different genetic backgrounds were analysed in the present study (Table 1). Chimeras 13.1, 13.2, 14.1, 15.1 and 15.2 were derived 
Table 1 Frequency of transgenic (parthenogenetic) nuclei in tissues of fetal parthenogenetic <-> fertilized chimeras in percent of total cell numbers. For most tissues, more than 1,000 nuclei on more than 1 section were counted (maximum number: 8815). Per- centage values derived from less than 1000 nuclei are italiazed, values derived from less than 300 nuclei are bold (n.d. tissue not found or not analysed)

\begin{tabular}{|c|c|c|c|c|c|c|c|c|c|c|c|c|c|}
\hline Day & 13.1 & 13.2 & $13.3^{a}$ & $13.4^{\mathrm{a}}$ & $13.5^{\mathrm{a}}$ & $13.6^{\mathrm{a}}$ & 14.1 & 15.1 & 15.2 & $17.1^{\mathrm{a}}$ & $17.2^{\mathrm{a}}$ & 17.3 & 18.1 \\
\hline Epidermis & n.d. & n.d. & n.d. & n.d. & n.d. & n.d. & n.d. & n.d. & n.d. & 21.1 & 36.6 & 82.2 & 13.7 \\
\hline Brain & 16.9 & 25.1 & 14.1 & 27.9 & 52.9 & 18.0 & 27.1 & 15.0 & 11.6 & 18.7 & 33.2 & 40.6 & 15.5 \\
\hline Cartilage & 14.4 & 9.7 & 10.7 & n.d. & n.d. & n.d. & 16.0 & 8.7 & 6.7 & 7.6 & 11.7 & 13.5 & 9.6 \\
\hline Skeletal muscle & 14.9 & 19.5 & 6.4 & 25.4 & 57.8 & 12.7 & 15.8 & 7.4 & 1.1 & 11.9 & 14.5 & 26.7 & 1.4 \\
\hline Heart & 19.5 & 9.1 & 9.8 & 38.7 & 14.1 & 9.6 & 18.4 & 13.3 & 4.0 & 6.0 & 12.4 & 24.7 & 5.3 \\
\hline Gut epithelium & 15.8 & 16.2 & 22.7 & 40.6 & 34.8 & 10.6 & 33.5 & 22.9 & 13.9 & 3.4 & 12.4 & 28.9 & 8.6 \\
\hline
\end{tabular}

a Only the foster mothers containing these fetuses had been injected with 5-bromo-2'-deoxyuridine (BrdU) before death

from pg $\left(\mathrm{F}_{1} \times 83\right)<->$ CFLP $\times$ CFLP aggregations; chimeras 13.3, 13.5, 13.6, 17.1 and 17.2 were of $\mathrm{pg}\left(\mathrm{F}_{1} \times 83\right)<-$ $>$ NMRI $\times$ NMRI genotype; chimeras 13.4 and 17.3 were made by pg $83<->$ NMRI $\times$ NMRI aggregations; and chimera 18.1 was of pg $83<->$ CFLP $\times$ CFLP genetic background. In addition, six postnatal chimeras aged between 21 and 101 days were analysed. Adult chimeras were derived from $\mathrm{pg}\left(\mathrm{F}_{1} \times 83\right)<->\mathrm{NMRI} \times \mathrm{F}_{1}$ (Nos. 1711 and 0202), pg $\left(\mathrm{F}_{1} \times 83\right)<->$ CFLP $\times$ CFLP (Nos. 215, 216, 217) and pg83 <-> (NMRIXBALB/c)F1 (No. 1903) aggregations.

\section{Distribution of pg cells in fetal tissues}

The contribution of pg cells to nine tissues of fetal chimeras was analysed quantitatively. These tissues were epidermis, brain, dorsal root ganglia (of ectodermal origin), cartilage (of either ectodermal or paraxial mesoderm origin), skeletal muscle (consisting of two cell lineages of mesodermal origin), heart, the smooth muscle and mesenchymal layers of the gut (all derived from lateral plate mesoderm), and the epithelium of the gut (derived from the endoderm). The results of the analysis are summarized in Table 1 and Fig. 1.

As expected from the randomness inherent in chimera formation, the mean pg contribution to the different chimeras varied. In most tissues of the chimeras analysed, however, pg-derived cells formed only a minor proportion of the total cell population. The distribution of pg cells to the different tissues was similar between chimeras. In the tissues of ectodermal origin, comparatively high levels of pg cells were regularly observed at all developmental stages, indicating that a significant loss of pg cells from these tissues during fetal development does not occur. In the other tissues, pg contribution tended to decrease with the advancing gestational age of chimeras.

In skeletal muscle, heart, cartilage, smooth muscle and intestinal epithelium of early post-midgestation chimeras, pg contribution reached levels close to the ones observed in the brain. However, with increasing age, the b Only smooth muscle and mesenchyme surrounding the intestinal epithelium were evaluated
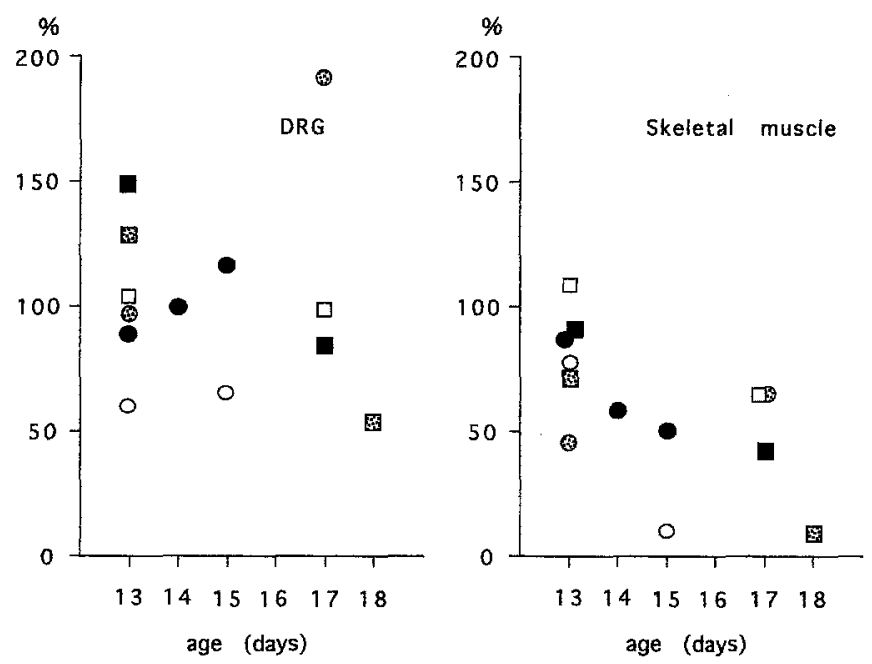

Fig. 1 The contribution of parthenogenetic cells to dorsal root ganglia $(D R G)$ and skeletal muscle of fetal chimeras is shown. For each chimera, values are normalized for parthenogenetic contribution determined in the brain $(100 \%)$. With advancing gestational age, the contribution to skeletal muscle decreases when compared with the brain. In contrast, parthenogenetic cells survive equally well in brain and in the dorsal root ganglia. Symbols represent individual chimeras. Day 13: $\bullet=13.1,0=13.2,=13.3, \mathbf{\square}=$ $13.4, \square=13.5$, 田 = 13.6; Day 14: $\bullet=14.1$; Day 15: $\bullet=15.1$, $=15.2$; Day 17: $\square=17.1, \boldsymbol{\square}=17.2, \Theta=17.3$; Day 18: 영 $=$ 18.1 .

levels of pg contribution in these tissues compared with the brain dropped considerably (Fig. 1). When the contribution of pg cells to different areas of cartilage was analysed, no striking differences were observed between mesoderm-derived cartilage of trunk and limbs and the ectoderm-derived cranial cartilage. For instance, in chimera $17.1,218 \mathrm{pg}$ nuclei were counted in a total of 3392 nuclei $(6.4 \%)$ in 10 different areas of neural crest-derived cartilage, e.g. the nasal bone (including the anterior part of the septum; see below) and the lower jaw. For comparison, in 13 different areas of sclerotome or lateral plate-derived cartilage, including ribs, vertebrae and limbs, 402 out of 4803 nuclei $(8.3 \%)$ carried the transgene. 
Table 2 BrdU incorporation into nuclei of parthenogenetically derived and normal cells in tissues of fetal parthenogenetic $<->$ fertilized aggregation chimeras. Data are presented as percentage values of BrdU-labelled nuclei/total number of nuclei for both parthenogenetic and wild-type cells in each tissue. Percentage values derived from more than 1,000 counted nuclei are not marked; values derived from less than 1,000 nuclei are italiazed, values derived from less than 300 nuclei are bold ( $P$ probability, pg parthenogenetic, $w t$ fertilized)

\begin{tabular}{|c|c|c|c|c|c|c|c|c|c|c|c|c|c|c|}
\hline Day & pg & wt & pg & wt & $\mathrm{pg}$ & wt & pg & wt & $\mathrm{pg}$ & wt & $\mathrm{pg}$ & wt & pg & wt \\
\hline 13.4 & n.d. & n.d. & 30.5 & 35.5 & 16.7 & 20.4 & n.d. & n.d. & 7.2 & 10.9 & 20.9 & 25.4 & n.d. & n.d. \\
\hline 13.5 & n.d. & n.d. & 25.7 & 26.0 & 7.8 & 11.4 & n.d. & n.d. & 4.5 & 26.9 & 14.3 & 19.2 & 23.9 & 29.2 \\
\hline 13.6 & n.d. & n.d. & 26.2 & 25.6 & 13.7 & 12.0 & n.d. & n.d. & 13.5 & 14.0 & 16.1 & 20.7 & 21.4 & 30.6 \\
\hline 17.2 & 25.5 & 30.8 & 13.8 & 15.0 & 5.7 & 6.3 & 4.3 & 10.3 & 7.9 & 16.8 & 14.8 & 15.8 & 30.4 & 38.8 \\
\hline $\mathrm{Pa}^{\mathrm{a}}$ & \multicolumn{2}{|c|}{$P=0.422$} & \multicolumn{2}{|c|}{$P=0.449$} & \multicolumn{2}{|c|}{$P=0.363$} & \multicolumn{2}{|c|}{$\mathrm{P}=0.001$} & \multicolumn{2}{|c|}{$\mathrm{P}=0.001$} & \multicolumn{2}{|c|}{$P=0.063$} & \multicolumn{2}{|c|}{$\mathrm{P}=0.016$} \\
\hline
\end{tabular}

a Statistical analysis was carried out using the Cochran-Mantel-Haenszel test that allowed pooling of proliferation data for a specific tissue from all chimeras of a given age group

\section{Proliferation of parthenogenetic cells}

Six pg $<->$ wt chimeras $(13.3,13.4,13.5,13.6,17.1$, 17.2) were removed from foster mothers that had been injected with BrdU prior to death. In seven tissues, the incorporation of BrdU into both normal and pg-derived nuclei was determined (Fig. 2L). The results of this analysis are summarized in Table 2.Three tissues of mainly ectodermal origin were evaluated, epidermis, brain and dorsal root ganglia. In these tissues, no consistent reduction in BrdU incorporation into pg nuclei, when compared with normal nuclei, was observed. Epidermis could not be analysed on day 13 of gestation, but in the two day-17 chimeras BrdU incorporation of pg and wt cells was not significantly different. Similar results were observed for dorsal root ganglia on both days 13 and 17 and for day- 17 brain. In the brain of the day-13 chimeras, however, the measured differences in BrdU incorporation between pg and wt nuclei were found to be significant.

The cartilage of the head is largely derived from the neural crest and thus is of ectodermal origin (Morriss and Thorogood 1978). The cartilage of the trunk is a derivative of the somitic sclerotome and thus is of paraxial mesodermal origin, whereas the limb cartilage is derived from the lateral plate mesoderm (Christ 1969). When these complications were neglected and all data were pooled, pg-derived cartilage cells showed decreased BrdU incorporation when compared with normal cells at both developmental stages.

In skeletal muscle of three day-13 chimeras, no striking difference between pg and wt proliferation was observed, although some variation in overall proliferation was found to exist between these chimeras. The exception was chimera 13.5 which exhibited a very low proportion of proliferating pg cells. At the same time, the proportion of proliferating wt cells was much higher than that observed in the other chimeras. The differences between pg and wt proliferation were not statistically significant either when 13.5 was included or excluded from the calculation.
Fig. 2 A A sagittal section through the cartilage ( $c a$ ) of the nasal septum of chimera 17.3 after DNA in situ hybridization (ISH). In the caudal area of this cartilage an almost complete absence of parthenogenetic (pg)-derived cells is obvious, but their number increases strikingly towards the tip of the nose. Numerous hybridization signals are also seen in some of the nasal epithelia shown (bar $100 \mu \mathrm{m}$, arrows see B and C). B, C Larger magnifications of two cartilage regions indicated on $\mathbf{A}$ by arrows. $\mathbf{B}$ The caudal region with very little pg contribution; $\mathbf{C}$ the rostral region with high contribution (bar $25 \mu \mathrm{m}$; DNA ISH). D A patch of pg-derived cells in the nasal cartilage $(c a)$ of the adult pg $\leftrightarrow$ wt chimera 1903 is shown, demonstrating that pg cells are perfectly able to form large patches of hyaline cartilage (bar $12.5 \mu \mathrm{m}$; DNA ISH and DAPI nuclear stain). E A small patch of pg cells in the hyaline cartilage (ca) of the trachea of chimera 1711 is shown (arrow). Positive cells were also observed in the endodermal epithelium (epi) of the trachea (arrowhead; bar $12.5 \mu \mathrm{m}$; DNA ISH and DAPI nuclear stain). F Skeletal muscle of chimera 13.1 after DNA ISH, anti-desmin immunohistochemistry (IHC) and 4,6diamidino-2-phenylindole DAPI nuclear staining is shown. While hybridization signals showing the presence of pg cells can be detected, no cells labelled by both DNA ISH and IHC are observed. This indicates that the pg cells present in this muscle blastema would have developed into intramuscular connective tissue, as myoblasts and myofibres express desmin at this stage (bar 25 $\mu \mathrm{m})$. G Desmin-positive muscle fibres of late gestation chimera 18.1 are shown. Five nuclei carrying a hybridization signal are visible in the muscle region, but the cytoplasm surrounding these nuclei is negative for desmin. This proves that these nuclei do not belong to the myogenic lineage (bar $25 \mu \mathrm{m}$; AP activity developed with new fuchsin - NF-as substrate, hence DNA ISH signals are red). H A nucleus labelled by DNA ISH (arrow) and situated inside a myotube can be detected in a muscle of chimera 18.1. This shows that pg cells are able to fuse with normal myoblasts to give rise to myotubes (bar $5 \mu \mathrm{m}$; DNA ISH using alkaline phosphataseNF and anti-desmin IHC using peroxidase-diaminobenzidine). I, J Consecutive sections stained by DNA ISH/DAPI (I) and HE haematoxylin-eosin ( $\mathbf{J})$ showing the presence of pg nuclei in skeletal muscle of chimera 1711. The morphology of the positive cell patch shows that these cells belong to the intramuscular connective tissue (bar $25 \mu \mathrm{m})$. K Positive cells situated in skeletal muscle of chimera 216 form the wall of a small blood vessel and surrounding mesenchyme. No nuclei situated inside the myotubes are labelled. (bar $12.5 \mu \mathrm{m}$; DNA ISH and DAPI nuclear stain). L Skeletal muscle of chimera 17.2 labelled by anti-BrdU IHC and DNA ISH. While numerous proliferating nuclei and pg nuclei can be detected in this section, the two labels rarely overlap (bar 12.5 $\mu \mathrm{m})$ 

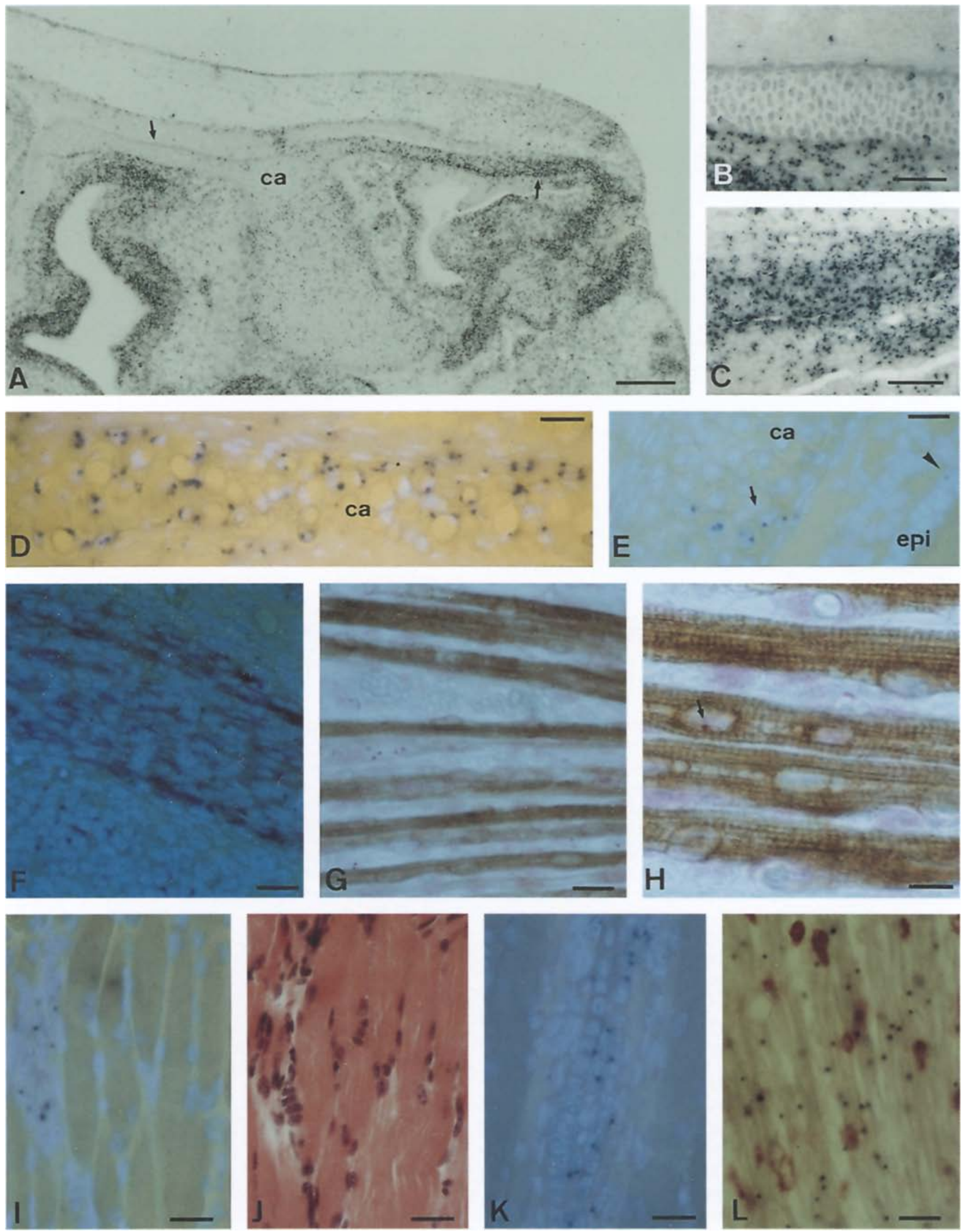
Table 3 Persistence of parthenogenetic cells in skeletal muscle and gastric epithelium of adult chimeras. Values are given as number of labelled nuclei per total number of nuclei (in parentheses percentage values)

\begin{tabular}{llllll}
\hline Chimera & 215 & 216 & 217 & 1711 & 0202 \\
\hline Skeletal muscle & $32 / 2061$ & $20 / 1619$ & $7 / 1500$ & $11 / 1402$ & $(0.8 \%)$ \\
& $(1.6 \%)$ & $(1.2 \%)$ & $(0.5 \%)$ & $44 / 195$ & $(2.6 \%)$ \\
Stomach & $35 / 360$ & $35 / 308$ & $13 / 291$ & $(21.0 \%)$ & $19 / 276$ \\
epitheliuma, b & $(9.7 \%)$ & $(11.4 \%)$ & $(4.5 \%)$ & $(6.9 \%)$ \\
\hline
\end{tabular}

a Data from Jägerbauer et al. (1992)

b Values show numbers of gastric glands that were counted; gastric glands are of monoclonal origin and are ultimately derived from a single progenitor cell

In the intestinal epithelium of three day-13 chimeras, no consistent difference between $\mathrm{pg}$ and $\mathrm{wt}$ proliferation was found. In both day-17 chimeras, however, pg cells incorporated significantly less BrdU than the neighbouring wt cells.

$\mathrm{Pg}$ cells in cartilage differentiation

In the analysis of pg contribution and proliferation in the cranial skeleton, a highly reproducible pattern of distribution of pg cells in the nasal bone emerged. It was found that in all pg $<->$ wt chimeras from day 13 onwards, pg cells tended to be present in much higher numbers in the anterior part of the nasal septum than in the posterior part. As the anterior part of the nasal bone remains a hyaline cartilage throughout the life of the mouse (Scott 1953), whereas ossification occurs in the posterior part during late fetal development (Fig. 2A, B, C), a causal relationship to the gradient of pg cell distribution was assumed. Also, random distribution of strain 83-derived normal and androgenetic cells was consistently observed in wt $83<->$ wt, wt $83 \rightarrow$ wt and ag $\rightarrow$ wt chimeras (not shown), indicating that this phenotype is specific for pg $<->$ wt chimeras. However, the non-random distribution of pg cells was visible well before the differentiation of the posterior cartilage was visible by morphological criteria. Accordingly, three different markers for cartilage differentiation were used, alcian blue staining, immunocytochemistry against type II collagen and mRNA ISH for IGF2. In the chimeras analysed, no expression gradient correlating with $\mathrm{pg}$ cell distribution was observed. In addition, no difference in collagen type II expression or alcian blue staining between pg and wt cells was obvious (not shown). IGF2, a growth factor that is encoded by a gene subject to genomic imprinting and expressed from the paternally derived allele only (DeChiara et al. 1991), exhibited no expression gradient between the rostral and caudal part of the nasal septum in a wt $<->$ wt chimera (not shown). Contribution of pg cells to the primary hyaline cartilage was observed in the nasal septum of 1903 (aged 81 days at death; Fig. 2D) and the tracheae of 1711, 0202 and 1903 (Fig. 2E).

\section{Pg cells in skeletal muscle differentiation}

To analyse in more detail the cellular composition of fetal skeletal muscle and the allocation of pg cells to dif- ferent lineages, sections of day 13 pg <-> wt chimeras were first subjected to IHC against desmin followed by ISH analysis. In muscle blastemas of two day-13 chimeras analysed (13.1 and 13.2, Table 1), desmin-positive structures corresponding to postmitotic myoblasts and early myotubes could easily be identified (Fig. 2F). An analysis of several muscle blastemas from both chimeras showed that desmin-positivity and presence of a hybridization signal coincided only rarely, e.g. most pg nuclei present in day-13 muscle were not associated with differentiated myoblasts or myotubes. In the muscle blastema shown in Fig. 1F no nucleus associated with a desminpositive structure exhibited a hybridization signal. A combined IHC and ISH analysis of two different skeletal muscle areas of the day-18 pg <-> wt chimera provided similar results. Here, only 1 out of $32(3.1 \%)$ and 7 out of $62(11.3 \%)$ nuclei which showed a hybridization signal were situated inside myotubes (Fig. $2 \mathrm{G}, \mathrm{H}$ ) and could thus be allocated to the myogenic lineage. The non-myogenic pg cells present in skeletal muscle of this chimera were found to belong to different cellular lineages, e.g. connective tissue and Schwann cells could be positively identified. Similar results were found in the other fetal pg $<>$ wt chimeras. In skeletal muscle of five adult chimeras that were analysed (Table 3 ), the majority of pg nuclei were found to belong to cells that could be unambiguously allocated to the intramuscular connective tissue and to small blood vessels (Fig. 2I, J, K). No nucleus could be allocated with any certainty to the muscle lineage proper.

\section{Discussions}

Previous studies have shown that chimeras carrying a pgderived cell lineage are viable (Fundele and Surani 1994) and that in such chimeras pg-derived cells may persist in numerous different tissues (Fundele et al. 1989, 1990; Nagy et al. 1989). However, the contribution of pg cells to most tissues is usually very low, although in gastrulation stage chimeras pg cells may be present in large numbers (Clarke et al. 1988; Thomson and Solter 1988). It has also been shown that selection against pg cells is strongly tissue specific (Fundele et al. 1989, 1990; Nagy et al. 1989). In general, in analysed tissues of ectodermal origin, e.g. brain and epidermis, pg-derived cells survive 
well into postnatal life. In other tissues, e.g. skeletal muscle, liver and pancreas, pg cells are subject to a stringent selection. It was therefore suggested that, in some tissues, pg cells may undergo a slowing down or even a cessation of cell proliferation after post-midgestation development (Surani et al. 1990a, b; Fundele et al. 1990). To test this hypothesis, we have analysed the distribution and proliferation of pg-derived cells in fetal aggregation chimeras using in situ detectable cell lineage (Lo 1986; Lo et al. 1987) and cell proliferation markers (Gratzner 1982). The feasibility of this approach has been demonstrated previously (Jägerbauer et al. 1992).

No systematic analysis of wt $<->$ wt control chimeras was performed for the present study. However, we and others (Nagy et al. 1989) have shown that the selective loss of pg cells is exclusive to the pg genotype. For wt83 <-> wt CFLP, wt $\left(83 \times \mathrm{F}_{1}\right)<->$ wt CFLP chimeras (analysed by ISH; Jägerbauer et al. 1992; Fundele, unpublished) and wt $\mathrm{F}_{1} \times \mathrm{F}_{1}<->$ CFLP chimeras (analysed by GPI-1 electrophoresis; Fundele et al. 1989, 1990) no indication of specific selection or allocation was ever observed at any developmental stage. The different strain combinations used in the present study for the production of the $\mathrm{pg}<->$ wt chimeras minimize the unlikely possibility that specific phenotypes are caused by strain background. In any case, a previous study has shown that the use of different mouse strains in the production of $\mathrm{pg}$ $<->$ wt or pg -> wt chimeras does not significantly change the fate of the pg cells (Fundele et al. 1991).

The results of quantitative analysis using the 83 transgene marker are in good agreement with previously published results obtained using the GPI-1 allozyme marker (Fundele et al. 1989, 1990, 1991; Nagy et al. 1989). Specifically, the best survival of pg cells during prenatal development was observed in the tissues of ectodermal origin, e.g. brain, dorsal root ganglia and epidermis. On the other hand, loss of pg cells from skeletal muscle was slower and less drastic than observed in a previous study (Fundele et al. 1990). At present we cannot explain this difference. It is, however, possible that different strain combinations may influence the behaviour of $\mathrm{pg}$ cells to some excent, as shown before (Fundele et al. 1991). In any case, the almost complete absence of pg-derived nuclei from adult skeletal muscle fibres was observed in all studies performed (Fundele et al. 1989, 1990, 1991; Nagy et al. 1989), including this one.

As expected from the good survival of pg cells in all tissues of ectodermal origin (except cranial cartilage), the proliferation of $\mathrm{pg}$ cells, as assessed by incorporation and immunohistochemical detection of BrdU, appears to be normal. A significantly decreased proliferation was found only in the brains of the day-13 chimeras. As BrdU labelling of pg cells was normal when compared with wt cells on day 17 of gestation, we assume that the day 13 finding does not reflect a real transient loss of pg neuroblast proliferation in early post-midgestation development.

When pg and wt proliferation in the intestinal epithelium were compared, a significant decrease with advanc- ing gestational age was observed. This decrease was associated with a highly significant drop in BrdU incorporation of pg cells between day 13 and 17 of gestation. The results of the proliferation analysis in $\mathrm{pg}<->$ wt chimeras seem to contradict previous findings that pg-derived stem cells are able to support normal gut morphogenesis during early postnatal life (Jägerbauer et al. 1992). It is, however, possible that this apparent discrepancy may be explained by the profound morphological changes that take place in the gut during perinatal development (Mathan et al. 1976). Among these changes are the transition from a stratified to a single-layer epithelium, the formation of folds and villi in the small intestine, the development of the crypts, and the spatial restriction of proliferating stem cells to the crypts (Mathan et al. 1976). It is feasible that pg cells that survive prenatal selection are no longer handicapped and can participate in the establishment of the adult pattern. However, there are indications that at least in some parts of the gut, pg cells are not able to sustain the normal cell proliferation and migration pattern (Jägerbauer et al. 1992).

The most impressive differences between pg and wt proliferation were observed in cartilage and skeletal muscle of the day 17 chimeras. Considering the well established fact that very few pg cells persist in adult skeletal muscle, the latter finding seems plausible. While the loss of pg cells from the skeletal muscle of chimeras seems to follow the pattern observed in other tissues, it is important to note that skeletal muscle is a composite tissue. In fact, the majority of nuclei present in an adult skeletal muscle do not belong to the myogenic lineage proper and a careful analysis of serial sections has shown that non-muscle cells may contribute up to $80 \%$ of the nuclei (Christ, unpublished). In addition, we have observed that by day 13 of gestation the majority of $\mathrm{pg}$ cells present in muscle blastemas do not belong to the myogenic lineage. Together, these observations indicate that the proliferating pg cells seen in skeletal muscle of day- 17 chimeras were mesenchymal and that loss of proliferative potential causes the demise of pg cells mostly from the intramuscular connective tissue. As we have shown in a previous study that pg cells contribute normally to the myotome of day-11 fetal chimeras (Fundele et al. 1994), it seems likely that the main loss of pg-derived cells from the myogenic lineage occurs between days 11 and 13 .

While a striking loss of proliferative potential was observed in fetal cartilage, pg cells were found to persist in the primary hyaline cartilage of adult chimeras. This apparent discrepancy is probably explained by the different behaviour of pg cells in anterior and posterior parts of the nasal septum. Here, pg cells survive in the rostral part that will remain a primary hyaline cartilage but not in the caudal part that will develop into bone. This suggests that factors involved specifically in ossification might be aberrantly expressed in pg cells.

In summary, our results show that loss of proliferative capacity is at least one cause for the loss of pg cells from those tissues where selection takes place. In this, pg cells 
show a strikingly different behaviour from ag cells which exhibit increased proliferation in ag $\rightarrow$ wt chimeras (Fundele, Li, Barton, Christ, Krause and Surani, unpublished). The mechanisms causing the decreased proliferation of $\mathrm{pg}$ cells are at present unknown. It is suggestive that in most of the tissues where pg cells do not persist, e.g. cartilage, skeletal muscle and liver (Fundele et al. 1989, 1990; Nagy et al. 1989), Igf2 is expressed at high levels. As only the paternally derived $I g f 2$ allele is active during normal development (DeChiara et al. 1991), it can be assumed that pg cells do not possess IGF2. In addition, pg cells probably overexpress $\operatorname{Igf} 2 r$ which maps to chromosome 17 and is expressed only from the maternal allele (Barlow et al. 1991). As absence of IGF2 alone does not cause a pronounced selective disadvantage in chimeras (Ferguson-Smith et al. 1991), it can be surmised that lack of $I g f 2$ expression and overexpression of $\operatorname{Ig} 2 \mathrm{r}$ combined are among the causes for the selective disadvantage of pg cell in some tissues. However, other known and unknown imprinted genes certainly play important roles in defining the developmental capacities of pg and ag cells.

Acknowledgements The authors are grateful to Dr. J. SchulteMönting for considerable help with the statistical analysis of our data. The technical assistance of A. Fraser and A. Herzfeld and the kind help given by Dr. E.-M. Jägerbauer, R. Krause, M. Reule and $\mathrm{U}$. Zechner at different stages of the work are also gratefully acknowledged. This work was supported by the Deutsche Forschungsgemeinschaft (grants Fu 188/2-2 and 188/3-1 to R. F.), the Wellcome Trust (grant 036481 to M. A. S.), by the University of Freiburg and by an ARC programme grant of the Deutsche Akademische Austauschdienst.

\section{References}

Anderegg C, Markert CL (1986) Successful rescue of microsurgically produced homozygous uniparental mouse embryos via production of aggregation chimeras. Proc Nat Acad Sci USA 83:6509-6513

Barlow DP, Stöger R, Herrmann BG, Saito K, Schweifer N (1991) The mouse insulin-like growth factor type-2 receptor is imprinted and closely linked to the Tme locus. Nature 349:84-87

Christ B (1969) Die Knorpelentstehung in den Wirbelanlagen. Experimentelle Untersuchungen an Hühnerembryonen. Anat Entwickl-Gesch 129:177-194

Clarke HJ, Varmuza S, Prideaux VR, Rossant J (1988) The developmental potential of parthenogenetically-derived cells in chimeric mouse embryos: Implications for action of imprinted genes. Development 104:175-182

DeChiara TM, Robertson EJ, Efstratiadis A (1991) Parental imprinting of the mouse insulin-like growth factor II gene. Cell 64:849-859

Ferguson-Smith AC, Cattanach BM, Barton SC, Beechey CV, Surani MA (1991) Embryological and molecular investigations of parental imprinting on mouse chromosome 7 . Nature 351:667-670

Fundele R, Bober E, Arnold HH, Grim M, Bender R, Wilting J, Christ B (1994) Early skeletal muscle development proceeds normally in parthenogenetic mouse embryos. Dev Biol $161: 30-36$

Fundele R, Howlett SK, Kothary R, Norris ML, Mills WE, Surani MA (1991) Developmental potential of parthenogenetic cells: Role of genotype-specific modifiers. Development 113:941946
Fundele RH, Norris ML, Barton SC, Fehlau M, Howlett SK, Mills WE, Surani MA (1990) Temporal and spatial selection against parthenogenetic cells during development of fetal chimeras. Development 108:203-211

Fundele R, Norris ML, Barton SC, Reik W, Surani MA (1989) Systematic elimination of parthenogenetic cells in mouse chimeras. Development 106:29-35

Fundele RH, Surani MA (1994) Experimental embryological analysis of genetic imprinting in mouse development. Dev Genet $15: 515-522$

Gratzner HG (1982) Monoclonal antibody to 5-bromo- and 5iododeoxyuridine: a new reagent for detection of DNA replication. Science 218:474-475

Jägerbauer E-M, Fraser A, Herbst EW, Kothary R, Fundele R (1992) Parthenogenetic stem cells in postnatal mouse chimeras. Development 116:95-102

Kaufman MH (1978) The experimental production of mammalian parthenogenetic embryos. In: Daniels JC (ed) Methods in mammalian reproduction. Academic Press, New York, pp 2147

Kossa J von (1901) Nachweis von Kalk. Beitr Pathol Anat Allg Pathol 29:163-183

Lo CW (1986) Localization of low abundance DNA sequences in tissue sections by in situ hybridization. J Cell Sci 81:143162

Lo CW, Coulling M, Kirby C (1987) Tracking of mouse cell lineage using microinjected DNA sequences: analyses using genomic Southern blotting and tissue-section in situ hybridizations. Differentiation 35:37-44

Mathan M, Moxey PC, Trier JS (1976) Morphogenesis of fetal rat duodenal villi. Am J Anat 146:73-92

Matthews DE, Farewell VT (1988) Using and understanding medical statistics. Karger, Basel London

Morriss GM, Thorogood P (1978) An approach to cranial neural crest cell migration and differentiation in mammalian embryos. In: Johnson MH (ed) Development in mammals. Elsevier North-Holland, Amsterdam, pp 363-411

Nagy A, Sass M, Markkula M (1989) Systematic non-uniform distribution of parthenogenetic cells in adult mouse chimeras. Development 106:321-324

Sasano Y, Mizoguchi I, Kagayama M, Shum L, Bringas P, Slavkin HC (1992) Distribution of Type I collagen, Type II collagen and PNA binding glycoconjugates during chondrogenesis of three distinct embryonic cartilages. Anat Embryol 186:205213

Scott JH (1953) The cartilage of the nasal septum. A contribution to the study of facial growth. Br Dent J 95:37-43

Stevens LC (1978) Totipotent cells of parthenogenetic origin in a chimaeric mouse. Nature 276:266-267

Solter D (1988) Differential imprinting and expression of maternal and paternal genomes. Annu Rev Genet 22:127-146

Surani MA, Barton SC, Howlett SK, Norris ML (1988) Influence of chromosomal determinants on development of androgenetic and parthenogenetic cells. Development 103:171-178

Surani MA, Allen ND, Barton SC, Fundele R, Howlett SK, Norris ML, Reik W (1990a) Developmental consequences of imprinting of parental chromosomes by DNA methylation. Phil Trans R Soc Lond B 326:313-327

Surani MA, Kothary R, Allen ND, Singh PB, Fundele R, Ferguson-Smith AC, Barton SC (1990b) Genome imprinting and development in the mouse. Development Supplement: 89-98

Thomson JA, Solter D (1988) The developmental fate of androgenetic, parthenogenetic, and gynogenetic cells in chimeric gastrulating mouse embryos. Genes Dev 2:1344-1351

Thomson JA, Solter D (1989) Chimeras between parthenogenetic or androgenetic blastomeres and normal embryos: allocation to the inner cell mass and trophectoderm. Dev Biol 131:580-583

Wilkinson DG, Green J (1990) In situ hybridisation and the threedimensional reconstruction of serial sections. In: Copp AJ, Cockroft DJ (eds) Postimplantation mammalian embryos: A practical approach. IRL Press, Oxford, pp 155-171 